\title{
Mathieu Vinken's Work on the Role of Connexin-related Signalling in Hepatic Homeostasis and its Relevance for Liver-based In vitro Modelling
} Mathieu Vinken*

Department of Toxicology, Faculty of Medicine and Pharmacy, Vrije Universiteit Brussel (VUB), Laarbeeklaan 103, B-1090 Brussels, Belgium

\begin{abstract}
Direct intercellular communication mediated by gap junctions constitutes a major regulatory platform in the control of hepatic homeostasis. Hepatocellular gap junctions are composed of two hemichannels of adjacent cells which are built up by connexin proteins, in casu Cx32. Dr. Mathieu Vinken, postdoctoral research fellow at the Department of Toxicology of the Free University Brussels-Belgium, was one of the first investigators to demonstrate that hepatic connexin expression is controlled by epigenetic mechanisms. In particular, he found that inhibitors of histone deacetylase enzymes enhance $\mathrm{Cx} 32$ production and gap junction activity in cultures of primary hepatocytes, a finding that is of importance for liver-based in vitro modelling. Dr. Mathieu Vinken's recent work is focussed on the elucidation of the role of connexin proteins and their channels in the hepatocyte life cycle. Specific attention is paid to apoptosis in this context, whereby it has yet been found that $\mathrm{C} \times 32$ hemichannels control the termination of induced cell death in cultures of primary hepatocytes. Overall, Dr. Mathieu Vinken's research can be considered as an important contribution to the field hepatic connexin physiology.
\end{abstract}

Keywords: Connexin; Hemichannel; Gap junction; Primary hepatocyte; In vitro modelling; Epigenetics; Histone modifications; Cell death; apoptosis; Hepatotoxicity

\section{Curriculum Vitae}

Mathieu Vinken (Figure 1) received his master's degree in pharmaceutical sciences at the Free University Brussels-Belgium (VUB) in 2001. In the same year, he step into a doctoral research project at the Department of Toxicology of the VUB under supervision of Prof. Dr. Vera Rogiers and Prof. Dr. Tamara Vanhaecke. During this research, entitled "effects of hydroxamate histone deacetylase inhibitors on the expression of connexins in primary cultures of rat hepatocytes", he was a doctoral research fellow of the Fund for Scientific Research FlandersBelgium (FWO). In 2006, he successfully completed and publically defended his doctoral research project and received the degree of doctor in pharmaceutical sciences. He continued his efforts in the field of connexin research in a subsequent postdoctoral project called "elucidation of the role of connexin proteins in the control of hepatocellular homeostasis: development of a hepatocyte-based in vitro model for preclinical pharmaco-toxicological research". At present, this research track, which is again supported by the FWO, is still being pursued. Dr. Vinken's work has yet resulted in as much as fifty scientific publications in peer-reviewed journals and books. He is co-inventor of two patent applications and has been an invited speaker on several international and world conferences. Dr. Vinken acts as a peer reviewer of many scientific journals and as an evaluator of project and grant applications for national and international research associations and agencies. He is

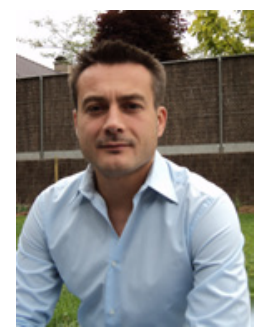

Figure 1: Mathieu Vinken, Ph.D, Pharm.D., Department of Toxicology, Faculty of Medicine and Pharmacy, Free University Brussels-Belgium. a frequently asked jury member for master and doctoral thesis defences and is co-organizer of a number of international scientific congresses and courses. Dr. Vinken is a regular member of five scientific societies in the area of toxicology and is as an executive board member of the European Society of Toxicology In vitro (ESTIV). He is co-founder of the FWO group of excellence called "connexin and pannexin channels: regulation, function and applications" and yet received two awards for his work. Dr. Vinken is currently a selected trainee in the European Toxicology Risk Assessment Training (TRISK) programme and has recently received the title of European Registered Toxicologist (ERT). $\mathrm{He}$ is actively involved in a number of European research projects in the Sixth (FP6) and Seventh (FP7) Framework Programme dealing with the development of in vitro assays for the toxicity testing of chemical compounds. Being an academic and given his background in pharmaceutical sciences, Dr. Vinken has been an assistant for the practical course "pharmaceutical technology: preparations for internal use" for a number of years. He also was involved in the organization of the "integrated practical course of biopharmacy" and "toxicology", and presently is responsible for the practical course "applied toxicology". Dr. Vinken is co-promoter of two doctoral theses in pharmaceutical sciences and has supervised several master thesis projects. Most of these dissertations serve as contributions to the Dr. Vinken's research on the roles of connexins and their channels in hepatic homeostasis and its relevance for liver-based in vitro modelling, as will be outlined in the following section.

*Corresponding author: Mathieu Vinken (Ph.D., Pharm.D.), Departmen of Toxicology, Faculty of Medicine and Pharmacy, Vrije Universiteit Brussel, Laarbeeklaan 103, B-1090 Brussels, Belgium, Tel: +32.2.477.45.87; Fax: +32.2.477.45.82; E-mail: mvinken@vub.ac.be

Received May 07, 2011; Accepted May 07, 2011; Published May 21, 2011

Citation: Vinken M (2011) Mathieu Vinken's Work on the Role of Connexin-related Signalling in Hepatic Homeostasis and its Relevance for Liver-based In vitro Modelling. Pharm Anal Acta 2:101e. doi:10.4172/2153-2435.1000101e

Copyright: (ㅇ 2011 Vinken M. This is an open-access article distributed under the terms of the Creative Commons Attribution License, which permits unrestricted use, distribution, and reproduction in any medium, provided the original author and source are credited. 
Citation: Vinken M (2011) Mathieu Vinken's Work on the Role of Connexin-related Signalling in Hepatic Homeostasis and its Relevance for Liverbased In vitro Modelling. Pharm Anal Acta 2:101e. doi:10.4172/2153-2435.1000101e

Page 2 of 5

\section{Research Achievements}

\section{General research context}

Cultures of primary hepatocytes are generally considered as the golden standard in the field of liver-based in vitro modelling since they provide a good reflection of the hepatic in vivo situation. In fact, these in vitro systems are abundantly used in several research fields, including pharmaco-toxicology and liver (patho) physiology. A major shortcoming of cultures of primary hepatocytes, however, is that they can only be used for short-term applications due to the occurrence of dedifferentiation, i.e. the progressive loss of the differentiated phenotype at the morphological level and at the functional level. Over the years, a number of strategies have been developed to counteract this dedifferentiation process. Such protocols are mainly based on mimicking the natural hepatocyte micro-environment in vitro, for instance by re-establishing cell-cell and cell-extracellular matrix contacts, but have only been of rather limited success [1-3]. In the last decade, the Department of Toxicology-VUB has explored a novel antidedifferentiation strategy for cultured hepatocytes based on chromatin remodelling. In this approach, histone deacetylase (HDAC) inhibitors are used as culture medium additives for primary hepatocytes. These epigenetic modifiers interfere with the chromatin structure and thereby alter transcriptional activity [2-6]. Research from the Department of Toxicology-VUB collectively showed that HDAC inhibitors suppress spontaneous cell death [7], induce cell cycle arrests [8,9] and concomitantly promote the (functional) differentiated phenotype in cultures of primary rat hepatocytes $[10,11]$. This is also associated with enhanced gap junctional communication between the hepatocytes.

Gap junctional intercellular communication (GJIC) denotes the passive flux of small and hydrophilic homeostasis regulators between cells. Gap junctions consist of two hemichannels of neighbouring cells, which in turn are built up by six connexin proteins (Figure 2). Connexin proteins are expressed in a cell-specific manner and are named after their molecular weight $[3,12-16]$. In the liver, hepatocytes produce
Cx32 (32 kDa), next to small amounts of Cx26 (26 kDa). By contrast, non-parenchymal liver cells, including Kupffer cells and stellate cells, mainly express $\mathrm{Cx} 43$ (43 kDa) [3,14]. As such, GJIC is considered as a key mechanism in the control of tissue homeostasis. It has indeed been shown that GJIC plays a crucial role in the performance of liver-specific functionality, particularly in xenobiotic biotransformation capacity and albumin secretory activity, as well as in hepatocyte proliferation $[3,14,15]$. The involvement of GJIC in (hepatocyte) apoptosis is less clear. In fact, the latter constitutes a relatively new research field, which has yet been complicated by the finding that structural precursors of gap junctions can affect tissue homeostasis by performing actions not related to GJIC. Thus, connexin hemichannels also foresee a pathway for communication, albeit between the intracellular compartment and the extracellular environment, while connexin proteins as such can directly or indirectly influence the production of homeostasis regulators independently of their channel activities. Furthermore, a novel set of connexin-like proteins, the so-called pannexins, have lately joined in as regulators of the homeostatic balance (Figure 3) $[12,16,17]$. Such atypical functions of connexins and their channels, specifically in the context of hepatocyte apoptosis, define Dr. Vinken's second area of interest, as will be clarified further in this paper.

\section{Study of epigenetic regulation of hepatocellular connexin expression}

Given its importance in the maintenance of tissue homeostasis, a well-orchestrated control of GJIC is critical. At the transcriptional level, the classical cis/trans machinery is known to act as a major gatekeeper of connexin expression [13]. In the last decades, it has become clear epigenetic determinants also drive gene transcription. Dr. Vinken was among the firsts to demonstrate that epigenetic mechanisms, particularly reversible histone acetylation, are essentially involved in the control of connexin production. In a pilot study, cultures of primary rat hepatocytes were exposed to the prototypical HDAC inhibitor Trichostatin A (TSA) and it was found that TSA increases Cx32 protein levels but negatively affects the Cx26 protein amounts.

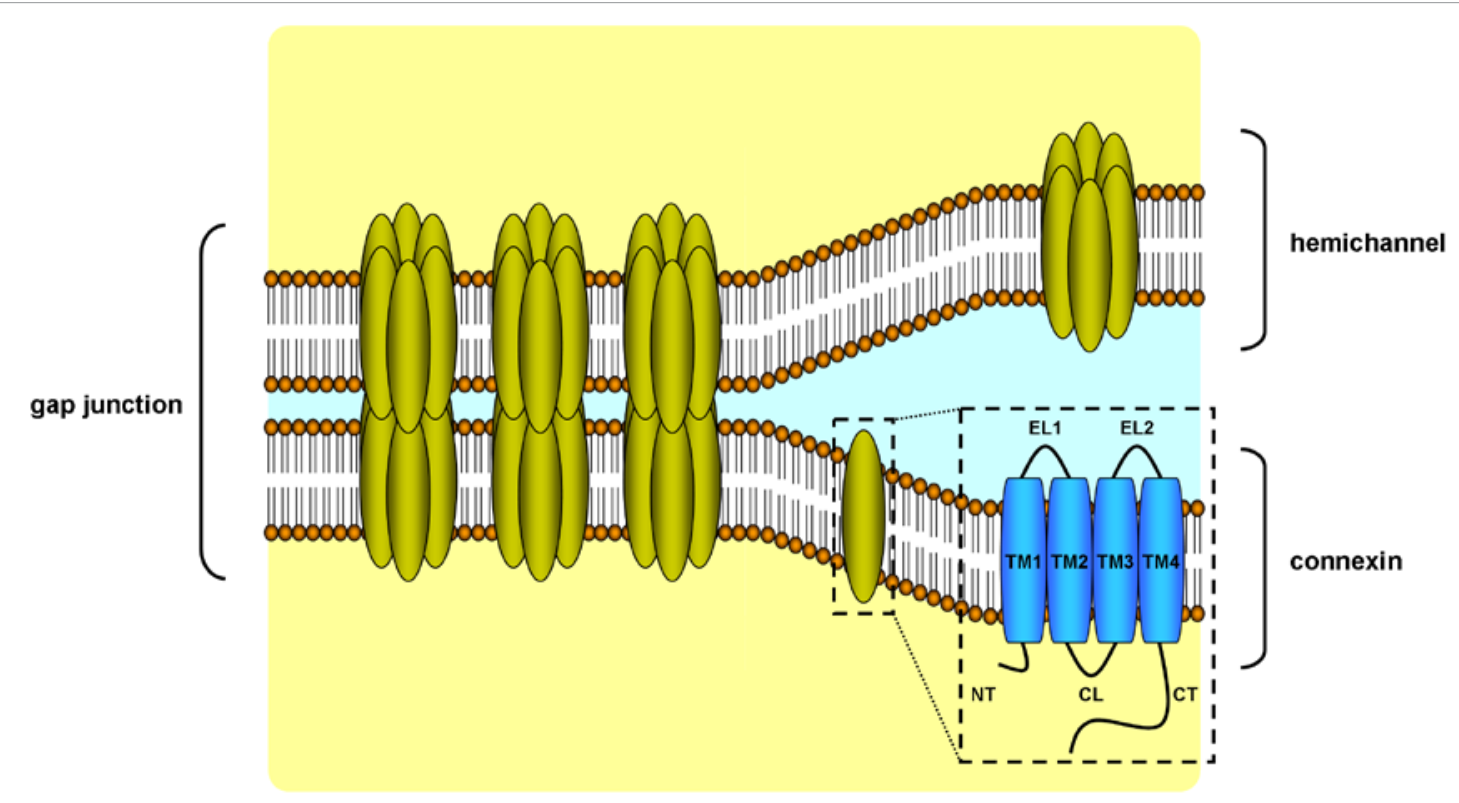

Figure 2: Molecular architecture of gap junctions. Gap junctions are grouped in plaques at the cell plasma membrane surface and are composed of twelve connexin proteins, organized as two hexameric hemichannels of two apposed cells. The connexin protein as such is organized as four membrane-spanning domains (TM1-4), two extracellular loops (EL1-2), one cytoplasmic loop (CL), one cytoplasmic amino tail (NT) and one cytoplasmic carboxy tail (CT). 


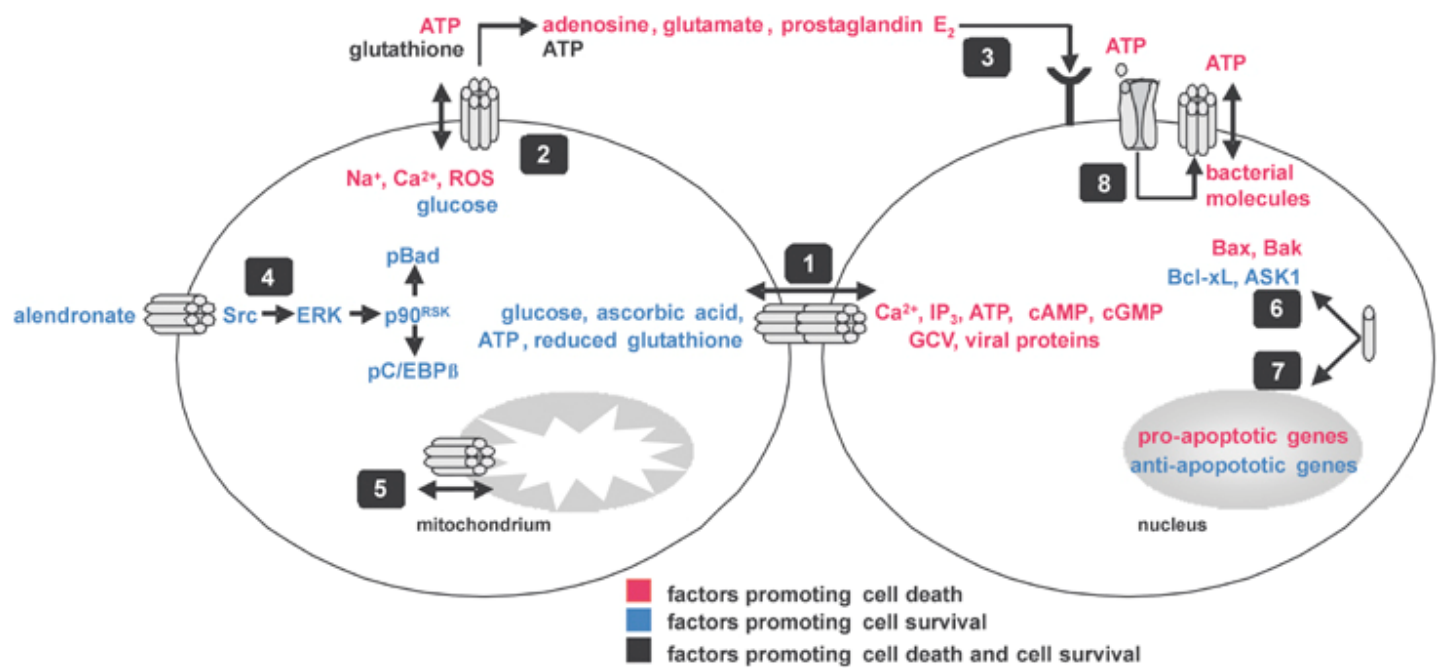

Figure 3: Connexin-related signaling in cell death. Connexins can affect the cell death process through a number of mechanisms, involving GJIC (1), hemichannels $(2-5)$ and connexins proteins as such $(6,7)$. Gap junctions can accommodate direct exchange of cell death and cell survival signals between cells (1). Hemichannels may contribute to cell death by four different mechanisms, namely by the entry of cell death or the loss of cell survival signals (2), through paracrine signaling of death or survival messengers (3), by hemichannel-mediated transmembrane signal transduction (4), or by affecting mitochondrial functioning (5). Connexin proteins as such can associate with cell death regulators (6) or influence the expression of these molecules (7). Hemichannels composed of pannexins may act as a permeabilization pore by itself or as a part of the $P_{2} X_{7} R$ death complex (8), allowing ATP to leave the cell or bacterial molecules to make their way into the cell. Although solid scientific data are currently not available, both processes might contribute to cell death. It should be noted that many of the first and second messengers depicted are not cell death or survival messengers per se, but rather substances that may lead to cell death or survival under specific conditions that are discussed in the text. (ASK1, apoptosis signal-regulating kinase 1; ATP, adenosine triphosphate; cAMP, cyclic adenosine monophosphate; cGMP, cyclic guanosine monophosphate; ERK, extracellular signalregulated kinase; GCV, ganciclovir; $\mathrm{IP}_{3}$, inositol trisphosphate; pBad, phosphorylated Bad; pC/EBP, phosphorylated CCAAT/enhancer-binding protein ; ROS, reactive oxygen species)

The latter was preferentially located in the cytosol of the cultured cells. TSA also promoted the appearance of $\mathrm{Cx} 43$ in the nuclear compartment of primary cultured hepatocytes. This connexin species is not expressed by hepatocytes in vivo, but becomes increasingly detectable upon cultivation of freshly isolated primary rat hepatocytes. Overall, the TSA-induced connexin modifications resulted in enhanced GJIC activity [18].

In a subsequent study, a similar experimental set-up was used, whereby the metabolically more stable TSA structural analogue 5-(4-dimethylaminobenzoyl)-aminovaleric acid hydroxamide (4- $\left.\mathrm{Me}_{2} \mathrm{~N}-\mathrm{BAVAH}\right)$ was added to the cell culture medium of the primary rat hepatocytes. With the exception of $\mathrm{Cx} 43$ protein levels, which were negatively affected by $4-\mathrm{Me}_{2} \mathrm{~N}-\mathrm{BAVAH}$, the findings were identical to those obtained with TSA. In the same study, the biological impact of $4-\mathrm{Me}_{2} \mathrm{~N}-\mathrm{BAVAH}$ on adherens junctions was investigated, being a group of cell contacts composed of cadherin-catenin complexes that mediate intercellular adhesion. Neither the expressions nor the cellular localizations of E-cadherin, $\beta$-catenin and $\gamma$-catenin were altered by 4-Me 2 N-BAVAH [19].

In summary, Dr. Vinken's studies show that HDAC inhibitors positively affect GJIC in cultures of primary rat hepatocytes. This finding further underscores the potential of the epigenetics-based strategy to counteract hepatocellular dedifferentiation in vitro, which is thoroughly explored by the Department of Toxicology-VUB. In addition, the differential effects of the HDAC inhibitors on connexin proteins in cultures of primary rat hepatocytes suggest distinct roles of the different connexin species in the control of hepatic homeostasis.

\section{Establishment and application of an in vitro model of liver cell death}

The Department of Toxicology-VUB has a long-standing expertise in the development and optimization of liver-based in vitro systems. Particular attention has been paid to the establishment of in vitro models of hepatocyte proliferation [20] and (functional) differentiation [10]. Dr. Vinken has been in charge of a project that was targeted towards the introduction of an in vitro system that enabled the study of the third cornerstone of hepatic homeostasis, namely cell death. In this research, emphasis was put on Fas-mediated cell death, which is a cell death mode that is of major physiological and pathological relevance, in casu in liver disease and hepatotoxicity. The developed in vitro model consists of freshly rat isolated hepatocytes, cultured in a monolayer configuration, that are exposed to a combination of Fas ligand and cycloheximide. This in vitro setting has been biochemically characterized by addressing a set of well-acknowledged cell death markers. In essence, the developed and fully characterized in vitro system allowed to monitor the entire course of Fas-mediated hepatocellular apoptotic cell death, going from early apoptosis towards the transition to a (secondary) necrotic phenotype [21].

The produced in vitro model of liver cell death was subsequently applied in a number of studies. In a first study, the effects of cell death on the expression of DNA methyltransferase (DNMT) isoenzymes were investigated. DNMT isoenzymes mediate DNA methylation, an epigenetic mechanism that acts in concert with histone (de)acetylation in gene transcriptional control. Similar to the hepatic in vivo situation, DNMT1, DNMT2 and DNMT3b could not be detected in cultures of primary rat hepatocytes, whereas relatively high levels of DNMT3a protein were observed. Upon induction of Fas-mediated cell death, a progressive decrease in DNMT3a protein amount was noticed which was preceded by parallel changes in DNMT3a mRNA production. This finding suggests the existence of an epigenetic signature of hepatocyte apoptosis [22].

In a second study, the outcome of Fas-mediated cell death on 
Citation: Vinken M (2011) Mathieu Vinken's Work on the Role of Connexin-related Signalling in Hepatic Homeostasis and its Relevance for Liverbased In vitro Modelling. Pharm Anal Acta 2:101e. doi:10.4172/2153-2435.1000101e

adherens junctions was investigated. Basically, it was found that E-cadherin expression gradually declined during the cell death process, whereas both $\beta$-catenin and $\gamma$-catenin were progressively degraded, yielding a number of proteolytic fragments. These results support the notion that dismantling of adherens junctions during hepatocyte apoptosis depends on proteolytic processing of its components [23].

\section{Elucidation of the role of connexins and their channels in cell death}

In the light of Dr. Vinken's interest in gap junction biology and physiology, most efforts were put on the application of the developed cell death model for investigating the fate of $\mathrm{Cx} 32$ and its channels in hepatocellular apoptosis. This research revealed that GJIC rapidly declines upon progression of cell death in cultures of primary rat hepatocytes, which is associated with a decay of the gap junctional Cx32 protein pool. Simultaneously, levels of newly synthesized Cx32 protein increase and gather in a hemichannel configuration. This becomes particularly evident towards the end stages of the cell death process and is not reflected at the transcriptional level. Both the silencing of $\mathrm{Cx} 32$ expression and the inhibition of $\mathrm{C} x 32$ hemichannel activity prior to cell death induction result in a delayed termination of the cell death response. Based on these findings, it was concluded that $\mathrm{C} \times 32$ hemichannels facilitate the apoptotic-to-necrotic transition during Fas-mediated cell death [24].

Dr. Vinken was also actively involved in a study whereby apoptosis was induced in rat glioma cells, stably transfected with $\mathrm{Cx} 43$, by in situ electroporation with cytochrome C. Work with various cell death markers, wild-type and Cx43-expressing cells, GJIC inhibitors and hemichannel inhibitors, and Cx43 gene silencing showed that gap junctions contribute to the spread of apoptosis in a zone next to where apoptosis was triggered, whereas hemichannels also promoted cell death beyond this area. It was concluded that $\mathrm{Cx} 43$ hemichannels, in concert with their gap junction counterparts, play a role in communicating cytochrome C-induced apoptotic cell death messages [25].

In an ongoing study conducted by Dr. Vinken, the relevance of induced Cx43 expression in cultures of primary rat hepatocytes is investigated. It is anticipated that $\mathrm{Cx} 43$ plays a role in spontaneous cell death in this in vitro setting, which is an inevitable consequence of the dedifferentiation process. To investigate the relevance of this hypothesis, a number of $\mathrm{Cx} 43$ inhibitor strategies has been developed and applied, and their outcome on cell death parameters is tested. The preliminary results support the assumption that $\mathrm{Cx} 43$ mediates the spontaneous cell death phenomenon in cultures of primary rat hepatocytes. Current experiments are focussed on the involvement of the different $\mathrm{Cx} 43$ channel types in this process as well as on the largescale outcome of the Cx43 inhibitor strategies on the hepatocellular phenotype by applying omics-based technologies.

\section{Conclusions and Perspectives}

Dr. Vinken's studies show that GJIC can be upregulated in cultures of primary rat hepatocytes by inhibition of HDAC enzymes, which not only fundamentally shows that gap junctions are subjective to epigenetic regulation, but which is also of utmost importance for the development of liver-based in vitro models that can be used for longterm research and screening purposes. Experiments are planned to investigate whether other determinants of the epigenome, including DNA methylation and microRNA-related mechanisms, are equally involved in GJIC control. Dr. Vinken's work also shows that connexin proteins and their channels fulfil critical functions in spontaneous and induced hepatocyte apoptosis, which as such contributes to the overall study of the relevance of connexin-related signalling in liver homeostasis. Future research will be focussed on the role of pannexinbased communication in the several aspects the hepatocyte life cycle.

\section{Acknowledgements}

Dr. Vinken is a postdoctoral research fellow of the FWO. The several studies presented in this paper were financially supported by grants of the FWO, the research council of the VUB and the European Union. Dr. Vinken is grateful to the past and present members of the Department of Toxicology-VUB for their contributions to his work. Dr. Vinken also wishes to express his gratitude to Prof. Dr. Paolo Meda (University of Geneva-Switzerland), Prof. Dr. James Kevin Chipman (University of Birmingham-United Kingdom), Prof. Dr. Geert Bultynck (University of Leuven-Belgium) and Prof. Dr. Luc Leybaert (University of Ghent-Belgium) for the fruitful collaboration

\section{References}

1. Elaut G, Henkens T, Papeleu P Snykers S, Vinken M (2006) Molecula mechanisms underlying the dedifferentiation process of isolated hepatocytes and their cultures. Curr Drug Metab 7: 629-660.

2. Papeleu $P$, Vanhaecke $T$, Rogiers $V(2006)$ Histone deacetylase inhibition: a differentiation therapy for cultured primary hepatocytes? Curr Enzym Inhib 2 : 91-104.

3. Vinken M, Papeleu P, Snykers S, De Rop E, Henkens T, et al. (2006) Involvement of cell junctions in hepatocyte culture functionality. Crit Rev Toxicol 36: 299-318.

4. Papeleu P, Vanhaecke T, Elaut G, Vinken M, Henkens T, et al. (2005) Differential effects of histone deacetylase inhibitors in tumor and normal cells: what is the toxicological relevance? Crit Rev Toxicol 35: 363-378.

5. Vanhaecke T, Papeleu P, Elaut G, Rogiers V (2004) Trichostatin A-like hydroxamate histone deacetylase inhibitors as therapeutic agents: toxicological point of view. Curr Med Chem 11: 1629-1643.

6. Vinken M, Peggy P, Vera R, Tamara V (2006) Histone deacetylase inhibitors as potent modulators of cellular contacts. Curr Drug Targets 7: 773-787.

7. Vanhaecke T, Henkens T, Kass GE, Rogiers V (2004) Effect of the histone deacetylase inhibitor trichostatin $A$ on spontaneous apoptosis in various types of adult rat hepatocyte cultures. Biochem Pharmacol 68: 753-760.

8. Papeleu P, Loyer P, Vanhaecke T, Elaut G, Geerts A, et al. (2003) Trichostatin $A$ induces differential cell cycle arrests but does not induce apoptosis in primary cultures of mitogen-stimulated rat hepatocytes. J Hepatol 39: 374-382.

9. Papeleu P, Wullaert A, Elaut G, Henkens T, Vinken M, et al. (2007) Inhibition of NF-kappaB activation by the histone deacetylase inhibitor 4-Me $2 \mathrm{~N}-\mathrm{BAVAH}$ induces an early $\mathrm{G} 1$ cell cycle arrest in primary hepatocytes. Cell Prolif 40 : 640-655.

10. Henkens T, Papeleu P, Elaut G, Vinken M, Rogiers V, et al. (2007) Trichostatin $A$, a critical factor in maintaining the functional differentiation of primary cultured rat hepatocytes. Toxicol Appl Pharmacol 218: 64-71.

11. Henkens T, Snykers S, Vinken M, Fraczek J, Lukaszuk A, et al. (2011) Preservation of hepatocellular functionality in cultures of primary rat hepatocytes upon exposure to 4-Me ${ }_{2} \mathrm{~N}-\mathrm{BAVAH}$, a hydroxamate-based HDAC inhibitor. Toxicol In Vitro 25: 100-109.

12. Vinken M, Vanhaecke T, Papeleu P, Snykers S, Henkens T, et al. (2006) Connexins and their channels in cell growth and cell death. Cell Signal 18 592-600.

13. Vinken M, De Rop E, Decrock E, De Vuyst E, Leybaert L, et al. (2009) Epigenetic regulation of gap junctional intercellular communication: more than a way to keep cells quiet? Biochim Biophys Acta 1795: 53-61.

14. Vinken M, Henkens T, De Rop E, Fraczek J, Vanhaecke T, et al. (2008) Biology and pathobiology of gap junctional channels in hepatocytes. Hepatology 47 1077-1088.

15. Vinken M, Doktorova T, Decrock E, Leybaert L, Vanhaecke T, et al. (2009) Gap junctional intercellular communication as a target for liver toxicity and carcinogenicity. Crit Rev Biochem Mol Biol 44: 201-222.

16. Vinken M, Decrock E, De Vuyst E, Ponsaerts R, D'hondt C, et al. (2011) Connexins: sensors and regulators of cell cycling. Biochim Biophys Acta 1815 13-25. 
Citation: Vinken M (2011) Mathieu Vinken's Work on the Role of Connexin-related Signalling in Hepatic Homeostasis and its Relevance for Liverbased In vitro Modelling. Pharm Anal Acta 2:101e. doi:10.4172/2153-2435.1000101e

Page 5 of 5

17. Decrock E, Vinken M, De Vuyst E, Krysko DV, D'Herde K, et al. (2009) Connexin-related signaling in cell death: to live or let die? Cell Death Differ 16: $524-536$

18. Vinken M, Henkens T, Vanhaecke T, Papeleu P, Geerts A, et al. (2006) Trichostatin A enhances gap junctional intercellular communication in primary cultures of adult rat hepatocytes. Toxicol Sci 91: 484-492

19. Vinken M, Henkens T, Snykers S, Lukaszuk A, Tourwé D, et al. (2007) The novel histone deacetylase inhibitor 4-Me $\mathrm{N}-\mathrm{BAVAH}$ differentially affects cell junctions between primary hepatocytes. Toxicology 236: 92-102.

20. Henkens T, Vinken M, Lukaszuk A, Tourwé D, Vanhaecke T, et al. (2008) Differential effects of hydroxamate histone deacetylase inhibitors on cellular functionality and gap junctions in primary cultures of mitogen-stimulated hepatocytes. Toxicol Lett 178: 37-43.

21. Vinken M, Decrock E, De Vuyst E, Leybaert L, Vanhaecke T, et al. (2009)
Biochemical characterisation of an in vitro model of hepatocellular apoptotic cell death. Altern Lab Anim 37: 209-218.

22. Vinken M, Snykers S, Fraczek J, Decrock E, Leybaert L, et al. (2009) DNA methyltransferase $3 a$ expression decreases during apoptosis in primary cultures of hepatocytes. Toxicol In Vitro 24: 445-451.

23. Vinken M, Decrock E, Leybaert L, Vanhaecke T, Rogiers V (2010) Proteolytic cleavage of adherens junction components during Fas-dependent cell death in primary cultures of rat hepatocytes. ALTEX 27: 151-157.

24. Vinken M, Decrock E, De Vuyst E, De Bock M, Vandenbroucke RE, et al. (2010) Connexin32 hemichannels contribute to the apoptotic-to-necrotic transition during Fas-mediated hepatocyte cell death. Cell Mol Life Sci 67: 907-918.

25. Decrock E, De Vuyst E, Vinken M, Van Moorhem M, Vranckx K, et al. (2009) Connexin 43 hemichannels contribute to the propagation of apoptotic cell death in a rat C6 glioma cell model. Cell Death Differ 16: 151-163. 